\title{
Neurodevelopmental Disabilities in Children with Retinopathy of Prematurity: A Report from India
}

\author{
Nagamani Beligere, $M D, M P H^{1^{*}}$, Vijayalakshmi Perumalsamy, $M D^{2}$, Jeyaseeli $H$ Flora, $M S^{3}$ and \\ Marla J Garska, MS $^{4}$
}

${ }^{1}$ Department of Pediatrics, University of Illinois at Chicago, II, USA

${ }^{2}$ Pediatric Ophthalmology, Aravind Eye Care System, Madurai Tamil Nadu, India

${ }^{3}$ Aravind Eye Care System, Madurai, Tamil Nadu, India

${ }^{4}$ Chicago Light House, Early Intervention Program, Chicago II, USA

*Corresponding author: Nagamani Beligere, MD, MPH, Department of Pediatrics, University of Illinois at Chicago, II, USA

\begin{abstract}
Background and objective: Retinopathy of prematurity (ROP) is a well-known complication of prematurity. However, the rates and prevalence of neurodevelopmental disabilities (NDD) among infants and children with a neonatal diagnosis ROP are not well known, particularly in low, and middle-income countries (LMIC).
\end{abstract}

Methods: We assessed the neurodevelopment, visual and neurological outcomes among children with a diagnosis of ROP referred to an ophthalmic center in India between 2014-2016. Neurodevelopmental status was assessed using skills inventory, for premature and primary school children who are blind and visually impaired. Visual and motor coordination status was assessed using Buketencia Developmental Test of Visual Motor Integration.

Results: Among the 152 infants with an ROP diagnosis, $104(68.4 \%)$ had NDDs and $48(32.6 \%)$ did not. Visual outcomes were abnormal in 55/104 (52.9\%), and normal in 49 $(47.1 \%)$. The rates of NDDs and visual impairments were highly correlated $(p<0.03)$, however, neither did the severity of ROP nor any neonatal variable correlated with NDDs. The types of NDDs were cognitive delay (23\%), motor delay $(24 \%)$, global delay $(16 \%)$, speech delay $(16 \%)$, and cerebral palsy $(12 \%)$.

Summary: In this large cohort of ROP infants from India, we found a high rate of NDDs. In nearly half of ROP infants who had normal visual outcomes had NDDs.

Conclusion: Premature infants with a diagnosis of ROP are at a high risk for developing NDDs, even when the visual outcomes are normal. The association of NDDs in ROP may have a common etiological basis, such as damage to the vascular and neuronal pathways during maturation. Our findings underscore the importance of ophthalmological and neurological follow-up and to undertake studies to explore the etiological basis for poor neurological and visual outcomes.

\section{Abbreviations}

ROP: Retinopathy of Prematurity, NDD: Neurodevelopmental Disabilities, LMIC: Low- and Middle-Income Countries, BW: Birth Weight, ELBW: Extremely Low Birth Weight, GA: Gestational Age, wks: Weeks

\section{Introduction}

Infants diagnosed as retinopathy of prematurity (ROP) in the neonatal period remain at high risk for long-term visual disabilities requiring regular screening and timely treatment. With the global improvement in neonatal care the survival rate of premature infants is increasing, so also in India. The increase in the incidence of ROP, a complication among the survivors [1] is well known. ROP is considered to be second most common cause for blindness. According to global report of 2008 [2], ROP is highly prevalent in all high, as well as in low and middle-income countries (LMICs) such as India and south Asian countries. Blencowe [3] estimated that during the years 2000-2009 nearly 20,000-30,000 children who developed ROP became blind or severe visually impaired worldwide. ROP in premature infants is known to develop both visual impairment and neurodevelopmental

Citation: Beligere N, Perumalsamy V, Flora JH, Garska MJ (2020) Neurodevelopmental Disabilities in Children with Retinopathy of Prematurity: A Report from India. Int J Ophthalmol Clin Res 7:114. doi. org/10.23937/2378-346X/1410114

Accepted: March 28, 2020: Published: March 30, 2020

Copyright: (C) 2020 Beligere N, et al. This is an open-access article distributed under the terms of the Creative Commons Attribution License, which permits unrestricted use, distribution, and reproduction in any medium, provided the original author and source are credited. 


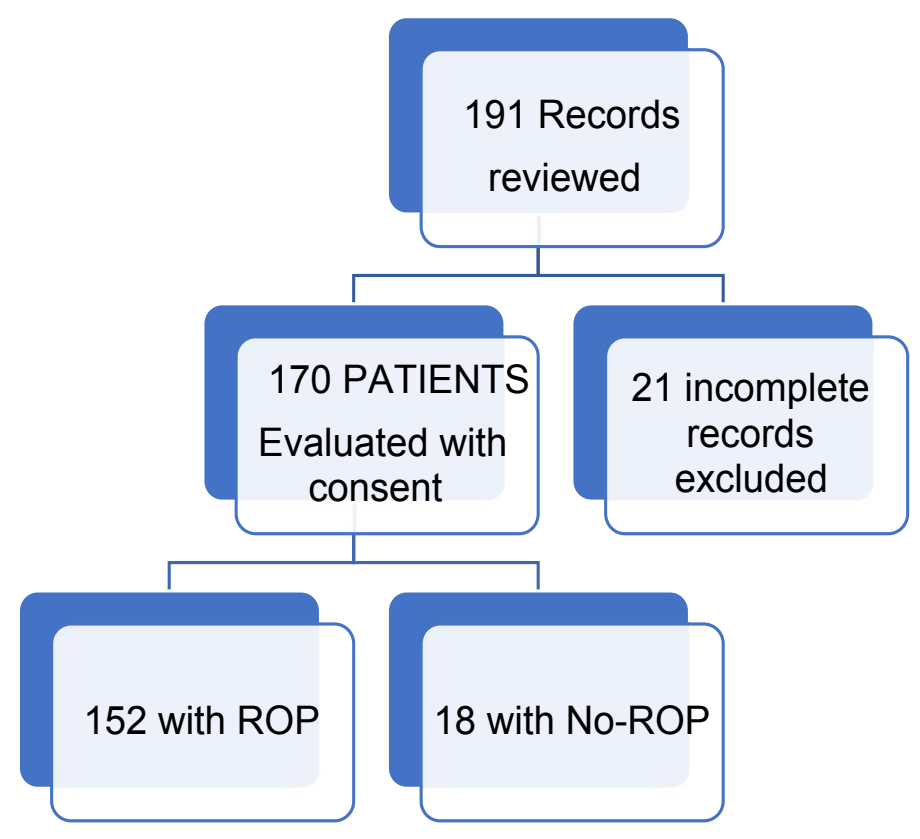

Figure 1: Flow diagram of patients' enrollment.

disabilities (NDDs). To understand the relationship of ROP and NDD, we launched a prospective cohort study in children with ROP referred for treatment and follow-up $t$ at Aravind Eye Care System (AECS), Madurai, India.

The objectives of this study were to assess the prevalence of NDDs in infants and children with a diagnosis of ROP and explore whether existence of ROP increases a risk for NDD. This may be useful for pediatricians the importance of screening and early treatment with supportive care.

\section{Background}

Aravind Eye Care System (AECS), Madurai, in the state of Tamil Nadu, India provides tertiary level of ophthalmic care for a population of 3 million people, in the District of Madurai. AECS, a post-graduate teaching and training institution in ophthalmology, conducts training and research opportunities for national and international students, and scholars. The center evaluates and treats nearly 400 children with ROP annually and has a program for long-term follow up for visual outcome and rehabilitation.

\section{Methods}

The investigator (NB) from University of Illinois at Chicago and an investigator from AECS (VP) initiated the collaborative study of neurodevelopmental assessment of children with ROP. Study was approved by the institutional review board of AECS. The study cohort included children referred to AECS for evaluation of ROP by pediatricians as well as parents. For this study, parents of 200 children born between 2009-2015 followed at AECS were contacted by phone or by mail. Parents were informed about the objectives of the study and written consent was ob- tained for NDD. Most mothers had at least $12^{\text {th }}$ standard education.

The primary inclusion criteria for this study was the diagnosis of ROP at the time of referral. Children with congenital malformation, genetic conditions and having, or neurological problems associated with, and IVH were excluded from the study.

The medical records of 191/200 infants and toddlers were retrieved, from available AECS. Nine records were unavailable due to parental concerns. 170 records were reviewed for detailed patient information of perinatal and neonatal history provided in the referral documents. 152 children had ROP and 18 had no ROP by visual examination (Figure 1).

The age of the infants at the time of referral for ocular examination by a pediatric ophthalmologist and vitreoretinal surgeons, were also recorded. Diagnosis of ROP stages according to the International classification [4] was recorded. Follow-up of ophthalmic evaluation was done on a weekly basis until a resolution was noted. The nature of ROP treatment (laser surgical management and/or Bevacizumab, Avastin) and ensuing complications, if any, were also recorded. The components of the follow-up ophthalmic evaluation included an estimation of the visual acuity, refractive errors; a need for further correction with corrective lenses; and intra-ocular pressure. Children were followed in the special clinic (the low-vision clinic) of the AECS for visual rehabilitation.

Comprehensive neurodevelopmental evaluation was performed by a developmental pediatrician (NB). Besides physical neurological examination, neurodevelopment was assessed using the specific tools known as the Skills Inventory, Oregon Project for Preschool and Primary School Children, who are Blind or visual- 
ly impaired $6^{\text {th }}$ edition [5]. The skills inventory comprised of functional evaluation of cognitive, gross motor, and fine motor, language and social and selfhelp skills, of children between the ages of 2 months $61 / 2$ years. Standardized scores were used to classify mild, moderate and severe neurodevelopmental disability. The developmental outcome was classified according to the assessment scores; normal when the scores were $>85$, mild when the scores were between 75-84; moderate when the scores were70-74; and severe when the scores were $<69$. Comprehensive Neurological evaluation was performed whenever severe delay was diagnosed. A diagnosis of cerebral palsy was made when the neurological assessment was abnormal in presence of hemiplegia, quadriplegia, diplegia, monoplegia, and/or athetotic movements. Children over 3 years of age were assessed for coordination defects and depth perception by VMI Test of Visual-Motor Integration, (VMI Test 2006 edition) [6] When indicated, children were recommended to receive the specialized therapeutic intervention in speech hearing, physical, and mental disability.

\section{Data Analysis}

The clinical, ophthalmic and developmental data was stored on computer for later analysis. Data were ana- lyzed by using Texas Instrument software STATA 11.1 (Texas USA). Mean and standard deviation, frequency, percentage were used to describe the summary data. Pearson Chi-square test or Fisher exact test was used to assess the association between the categorical variables. Wilcox test was used for non-parametric values. Both tests provided consistent results through out. Univariate analysis of association of NDD, with reference to GA, BW and perinatal variables did not show any significance.

\section{Results}

One hundred and ninety-one children referred for confirming the diagnosis of ROP were enrolled for neuro developmental assessment during January 2014-March 2016. Consents were available for 170/191 children, in whom we completed all evaluations. Twenty-one infants with incomplete records were excluded from further analyses. Thus, we had 152 children with the diagnosis of ROP, and 18 children who did not have ROP but were followed in the eye clinic for other ophthalmic reasons (Figure 1). The baseline characteristics of BW and GA and Perinatal variables of the 152 infants are provided in Table 1.

Forty nine percent were born vaginally and 51 percent by c section. $57 \%$ were males and $43 \%$ females.

Table 1: Neurological outcome in relation to GA and BW and perinatal variables.

\begin{tabular}{|c|c|c|c|c|}
\hline Variables & Developmental Outcome NDD Normal & NDD Abnormal & Total & p-value \\
\hline & & & 152 & \\
\hline All Infants (152) & 48 & 104 & & \\
\hline \multicolumn{5}{|l|}{ Gender } \\
\hline Males (86) & 24 & 62 & 86 & 0.266 \\
\hline Females (66) & 24 & 42 & 66 & \\
\hline \multicolumn{5}{|c|}{ Ages < 1-> 5 yrs (152) } \\
\hline$<1 \mathrm{yr}(15)$ & 5 & 10 & & \\
\hline $1-2$ yrs (48) & 14 & 34 & & \\
\hline $2-3$ yrs $(40)$ & 10 & 30 & & \\
\hline 3-4 Yrs (27) & 12 & 15 & & \\
\hline $4-5$ yrs $(10)$ & 2 & 8 & & \\
\hline$>5$ yrs $(12)$ & 5 & 7 & & \\
\hline$<2$ yrs $(63)$ & 19 & 44 & & 0.751 \\
\hline$>2$ yrs (89) & 29 & 60 & & \\
\hline GA in wks (152) & & & 152 & \\
\hline 25-28 wks (23) & 8 & 15 & 23 & \\
\hline $29-32$ wks (70) & 19 & 51 & 70 & \\
\hline 33-35 wks (45) & 16 & 29 & 45 & \\
\hline > 35 wks (14) & 5 & 9 & 14 & \\
\hline
\end{tabular}




\begin{tabular}{|c|c|c|c|c|}
\hline GA < 32 wks $(93)$ & 27 & 66 & 93 & 0.396 \\
\hline GA > 32 wks (59) & 21 & 38 & 59 & \\
\hline BW < 1500 g (89) & 27 & 62 & 89 & \\
\hline BW > 1500-2500 g (63) & 21 & 42 & 63 & 0.695 \\
\hline Pregnancy complications ( ) & & & 152 & \\
\hline Yes (113) & 27 & 66 & 113 & \\
\hline No (39) & 9 & 30 & 39 & \\
\hline Birth Type & & & 152 & \\
\hline Singletons (97) & 31 & 66 & 97 & 0.894 \\
\hline Multiple (55) & 17 & 38 & 55 & \\
\hline \multicolumn{5}{|l|}{ Mode of Delivery } \\
\hline CS (77) & 26 & 51 & 77 & 0.557 \\
\hline NSVD (75) & 22 & 53 & 75 & \\
\hline RDS & & & 129 & \\
\hline Yes (129) & 40 & 89 & 129 & 0.72 \\
\hline No (23) & 8 & 15 & 23 & \\
\hline $\begin{array}{l}\mathrm{RDS} \text {, and } \mathrm{O}_{2} \text { therapy plus } \\
\text { assisted ventilation }\end{array}$ & & & 152 & \\
\hline Yes (115) & 35 & 80 & 115 & 0.593 \\
\hline no & 13 & 24 & 37 & \\
\hline Severe Neurologic deficits & & & & 0.506 \\
\hline Yes (10) & 2 & 8 & 10 & \\
\hline No (142) & 46 & 96 & 142 & \\
\hline Seizures yes (44) & 9 & 35 & 44 & 0.060 \\
\hline No Seizures & 46 & 69 & 98 & \\
\hline MRI & & & 152 & \\
\hline Yes (11) & 0 & 11 & 11 & 0.017 \\
\hline NO (141) & 48 & 93 & 141 & \\
\hline
\end{tabular}

Table 2: Neurologic outcome and Visual outcome in relation to treatment.

\begin{tabular}{|c|c|c|c|c|}
\hline Visual Outcome & $\begin{array}{l}\text { NO NDD } \\
\text { Normal development }\end{array}$ & $\begin{array}{l}\text { ANY NDD Developmental } \\
\text { outcome }\end{array}$ & Total & p-value \\
\hline Visual impairment & & & 152 & \\
\hline Yes & 13 & 55 & 68 & 0.003 \\
\hline no & 35 & 49 & 84 & \\
\hline Laser treatment & & & 152 & 0.0243 \\
\hline yes & 37 & 68 & 105 & \\
\hline No & 11 & 36 & 47 & \\
\hline Laser + Bevacizumab treatment & & & 25 & \\
\hline Yes & 11 & 14 & 25 & 0.144 \\
\hline No & 37 & 90 & 127 & \\
\hline
\end{tabular}


Gestational age (GA) ranged from 25-40 wks. With median of 32 wks. Ninety-three children were $<32$ wks. 59 children were $>32$ wks. Birth weight ranged, from 545 g-2915 g with median BW of 1420 g. 59\% [89n] of the children were $<1500 \mathrm{~g}$, 58 [39\%] were $>1500 \mathrm{~g}-2500 \mathrm{~g}$, only $4 \mathrm{n}[3 \%$ ] children were $>2500$ g. $63.8 \%$ [97n] were singletons, $30.9 \%[47 n]$ were twins, and 5.3\% [8n] triplets. $85 \%[129 n$ ] of infants had diagnosis of RDS.76.3\% [116n] of them received $\mathrm{O}_{2}$ therapy and required ventilation. Surfactant was used in $16.5 \%$ [25n] children $28.9 \%$ [ $44 \mathrm{n}$ ] had a history of seizures. EEG abnormality was noted in $9.9 \%$ [ $15 \mathrm{n}$ ], MRI was performed in $7.2 \%$ [11n] of the ventilated infants. The length of hospital stays ranged from 5-90 days with median of 23 days. Univariate analysis of association of NDD with perinatal variables of GA, BW, RDS, Apgar score, mode of delivery $\mathrm{O} 2$ inhalation did not show any statistical significance. Table 2 includes, ROP treatment with laser surgery in $64 \%$ [ $194 \mathrm{n}$ ] eyes, and 14.9\% [45n] with laser and bevacizumab. The Visual outcome and NDD outcome are also included. Poor Visual outcome was associated with poor developmental outcome.

\section{Neurodevelopmental outcomes in children with ROP}

Neurodevelopmental assessment was done in 152 children. Age at assessment ranged from 3 months to 105 months with median age of 28.5 months. Neurodevelopmental abnormalities were found in 104 (68.4\%) children and no neurological abnormalities were diagnosed in $31.6 \%$ [48]. Among those with NDDs, $24.3 \%$ [37n] children had mild delay, $14.5 \%$ [22] children had moderate delay and $29.6 \%$ [ $45 \mathrm{n}$ ] had severe delay. Among the children who had seizures, 80\% [35] had abnormal neurodevelopment. All 11 Children with abnormality of MRI findings during follow up, were found to have developmental abnormalities.

Bilateral ROP was diagnosed in 150 infants (300eyes) and 2 had unilateral ROP. One-hundred ninety eyes $64 \%$ [194] eyes were treated with laser surgery and $14.9 \%$
[45] were treated with laser surgery as well as bevacizumab (Avastin). Among infants with normal visual outcomes (with corrective lenses) 58.3\% [49] were found to have abnormal neurodevelopment. These were mild to moderate NDD. Two children with unilateral ROP had normal neurological development.

Seventy-four children who were between the ages of 30 to 60 months, were also evaluated for Visual Motor Integration test (VMI). We found 34 (50\%) of them to have visual impairment with coordination defect and depth perception abnormalities which interfered with developmental outcome.

Among 126 infants with ROP stages 1, 2, and 3 we found $34.9 \%$ [44] with abnormal visual outcomes, whereas 23 of 24 infants with ROP stages 4 or greater had abnormal visual outcome. There was no significant association between the Stages of ROP and NDD ( $p=$ 0.079). But there was significant association of ROP with Visual outcome $(p<0.001)$ Table 3 . Only $47 \%$ of the children who had refractive errors were treated with corrective lenses.

The nature of NDDs varied, in children with corrected vision. $81 \%$ had visual motor coordination and depth perception, defects. When we analyzed the Visually impaired children with developmental outcome visually impaired children had significant abnormality. The association of visual outcome to developmental outcome was significant $(p<0.003)$ Table 4.

\section{Neurologic outcome with ROP and without ROP (Table 5)}

Neurologic Abnormality with ROP: Major neurodevelopmental abnormal findings is shown in the Table 5. Neurologic impairments included cerebral palsy $11.5 \%$ [12n], cognitive impairment $23.1 \%$ [24n], motor delay in $23 \%$ [24] and global delay $15.3 \%$ [ $16 n$ ] speech impairment, $15.3 \%$ [16n] and hearing difficulties. Social, emotional and self-help skills were delayed in most children.

Table 3: ROP stages and visual outcome.

\begin{tabular}{|l|l|l|l|l|}
\hline \multirow{2}{*}{ ROP stage } & Visual outcome & Total & p-value \\
\cline { 1 - 3 } & Normal & Abnormal & & \\
\hline Stage 1, 2, 3 \& APROP & $65.1 \%[82]$ & $34.9 \%[44]$ & 126 & $<0.001$ \\
\hline Stage 4 \& 5 & $4.2 \%[1]$ & $95.8 \%[23]$ & 24 & - \\
\hline Total & 83 & 67 & 152 & \\
\hline
\end{tabular}

Number of children in each group is shown in [ ]

Table 4: Visual impairment and Developmental outcome.

\begin{tabular}{|l|l|l|l|l|}
\hline \multirow{2}{*}{ Visually impaired } & \multicolumn{2}{|l|}{ Developmental outcome } & Total & p-value \\
\cline { 2 - 4 } & Normal & Abnormal & & \\
\hline Yes & $13(19.1)$ & $55(80.9)$ & 68 & 0.003 \\
\hline No & $35(41.7)$ & $49(58.3)$ & 84 & - \\
\hline Total & 48 & 104 & 152 & \\
\hline
\end{tabular}


Table 5: Neuro Developmental Disabilities in ROP and No-ROP.

\begin{tabular}{|l|l|l|l|l|l|l|l|}
\hline Group & CP & Motor delay & Cognitive delay & Global delay & Speech & Hearing & Blind \\
\hline ROP [104] & $12 \%[12]$ & $23 \%[24]$ & $22.1 \%[23]$ & $15.3 \%[16]$ & $15.3 \%[16]$ & $3.8 \%[4]$ & $8.6 \%[9]$ \\
\hline NO-ROP [15] & $6.6 \%[1]$ & - & $33.0 \%[5]$ & $6.6 \%[1]$ & $26.6 \%[4]$ & $13.3 \%[2]$ & $13.3 \%[2]$ \\
\hline
\end{tabular}

Number of children in Each group is shown in [ ]

\section{Neurodevelopmental outcomes in children with no ROP: Table 5}

There were 18 children with no-ROP. Birth weight ranged from $500 \mathrm{~g}$ to $1500 \mathrm{~g}$, GA ranged from 25-34 weeks. All were treated for RDS and ventilated. Of these $72.2 \%$ children developed visual impairment, 2 were blind. Visual impairment was due to causes other than ROP. In this group of children evaluation showed normal vision and development only in $17 \%$ [3n]. Rest $83 \%$ [15n] were noted to have neurodevelopmental abnormalities. The disabilities were mild delay in $62 \%$, moderate delay in $11 \%$. Severe deficit with cerebral palsy in $6.6 \%[1 \mathrm{n}]$ and global delay in $6.6 \%$ [1n], cognitive delay in $33.0 \%$ [ $5 \mathrm{n}$ ], speech delay in $26.6 \%$ [ $4 \mathrm{n}$ ] and hearing impairment in $13.3 \%$ [2n] and Blindness in $13.3 \% \%$ [2n] were noted.

\section{Discussion}

It is increasingly recognized that ROP is an emerging problem with increasing survival rates for preterm infant world over, including LMICs [1-3]. The early eye health screening and treatment of premature infants are lacking in LMICs due to poor socioeconomic conditions and lack of public awareness, which is similar to the reports of Vartanian [7] even in high-income countries such as US. In spite of these deficiencies, there are several reports on the incidence of ROP from LMICs $[2,3]$. In addition to visual impairments, infants with ROP are prone to develop neurodevelopmental delays [3] which is not well known in LMICs.

Gilbert, et al. in 1997 evaluated 4121 children in blind schools from 23 LMIC countries for visual acuity [1]. They found that ROP was one of the main etiologies for blindness. A later study they concluded that prevalence of ROP depends on neonatal mortality rates (NMR) [2]. Bas AY, et al. [8] reported ROP incidence from 49 neonatal ICUs in Turkey. ROP of any stage was noted in $56 \%$ of $<1000 \mathrm{~g}$, and $14.5 \%$ in infants of $1500-2000 \mathrm{~g}$. Incidence of severe ROP was in $8 \%$ of infants with $<1500 \mathrm{~g} \mathrm{BW}$, and $0.6 \%$ in infants $>1500 \mathrm{~g}$. In a later study of 69 NICUs [9] they found ROP of some stage in $81 \%$ of infants $<32$ wks. GA vs. $19 \%$ in $>32$ wk GA group. The study shows that more mature infants were also at risk of developing ROP in their population.

Several investigators from India also reported similar incidence in respective study populations. Rekha, et al. [10] reported the incidence of ROP in $73 \%$ in $<1000 \mathrm{~g}$, $60 \%$ in GA $<32$ wks. Recent data from India show much lower rates of ROP. Choudhry, et al. [11] reported 22\%
ROP in $<1500 \mathrm{~g}$ infants and no ROP was found in $>2000$ g Babies. Vinekar, et al. [12] evaluated ROP in 1250$2750 \mathrm{~g}$ infants and found $45 \%$ of those had threshold ROP. A recent study by Maheshwari, et al. [13] reported incidence of ROP as $18.4 \%$ in < $1750 \mathrm{~g}$. ROP was found in both decreasing birth weight as well as in larger birth weight. ROP in decreasing in lower birth weight suggested improved survival, neonatal and perinatal care.

Most reports of ROP [14-17] described primarily the pathologic changes in retina which leads to myopia with age. Cook, et al. [14] Choi, et al. [15] showed that myopia in ROP is the result of decrease in the depth of anterior chamber and high refractive power of the lens. The observed degree of myopia increased from 6 months to 3 yrs of age, with no increase between 3 to 6 years, Tasman, et al. also [16] reported that myopia associated with ROP continues to be a problem into adult life. Kaur, et al. [17] found $75 \%$ of refractive errors were due to severe ROP in $>1700 \mathrm{~g}$ after laser surgery. In spite of the structural correction, $6 \%$ of children have unexplained visual deficiency. Visual acuity may be the determining factor for functional outcome. We found that children with visual correction also found to have NDD. In our study the Stage of ROP to visual outcome was significant (<0.001) Table 3.

Kaminar, et al. [18] first recognized the relationship of visual impairment as detrimental to child's development and learning ability. Msall [19] reported NDD in institutionalized infants who were $<1250 \mathrm{~g} \mathrm{BW}$ at 5.5 years of age with threshold ROP treated with cryotherapy. They found severe NDD and cognitive disability in $75 \%$ with poor visual acuity compared to children with better visual acuity even in one eye $(p<0.001)$. Cooke, et al. [20] reported neurodevelopmental abnormalities, in ROP children < 32 wk GA. Visual impairments were associated with NDD with significant motor and cognitive delay, very low verbal IQ at 7 years of age. They concluded that these abnormalities may be due to abnormal cortical development rather than prenatally acquired insult. Schmidt, et al. [21] studied 910 surviving ELBW infants born between the 1996-98, using discharge ultrasound, and the discharge diagnosis of BPD, NEC and ROP. Risk of death or neuro developmental disability was higher at 18 months of age in ROP children. Later study Schmidt, et al. [22] found that severe ROP children at five years age had four times higher neurodevelopmental disabilities. They attributed cognitive delay to maternal race. Laverson, et al. [23] reported studies from Norway and concluded that the NDD outcome in 22-27 wk GA and 500-999 g BW was based on the ma- 
ternal and neonatal factors. Blencowe, et al. [3] in an analysis of 10 studies reported association of ROP and NDD found, $55 \%$ of visually affected ROP infants had NDD. Goyen [24] described the finer motor coordination defects in children with ROP. VMI test revealed that these children had lower scores compared to children without ROP regardless of stage of ROP. We also report similar findings with Visual Motor coordination defects.

We [25] previously reported that the disabilities were higher with severe ROP in Zone-1 involvement. Association of visual impairment to NDD was significant $(p<0.006)$. The study showed that when degree of ROP was greater than stage-3, involving Zone-1, severity of the NDD was $89.5 \%$, when Zone- 3 was affected NDD was $42.1 \%$ and when zone- 2 was affected neurodevelopmental abnormality was in $69.4 \%$ ( $p-<0.007)$. Children with ROP, in spite of visual correction were found to have difficulties with visual motor coordination and depth perception. These defects are hindrance to learning in school, limiting the environmental experience for children. This suggests ROP in premature children, continued to be a factor for neurodevelopment abnormality, regardless of early treatment.

The observed association of NDD in ROP children can be further explained based on white matter maturational changes. Glass, et al. [26] studied the relationship of white matter maturation with developmental outcome in infants born at 24-28 wk GA, with severe ROP and infants who had no ROP. White matter maturation was assessed by mean fractional anisotropy (FA) using $\mathrm{MRI}$ in seven predefined regions at 7 years of age, found that severe ROP was associated with poor posterior optic radiation in both internal and external capsule. They found lower cognitive and motor scores in severe ROP. Sveinsdottir, et al. [27] showed reduced brain volume and impaired development in any stage of ROP. They concluded that there is a common neuronal pathway of neuronal and neurovascular development in the brain as well as retina, which might explain the association of ROP and NDD.

Rainey, et al. [28] observed that. The low vision in children with ROP affects not only the visual development but also the physical, psychosocial social development. Children with ROP also have difficulty in postural awareness, movements of hand -eye coordination. They also have difficulty in understanding the meaning of the words thus affecting the social skills and poor self-help. Further these children develop limited peer relationship and develop ambivalent attachment since they are not able to recognize visual cues or facial expression of others. The interaction is limited. Similar studies by Tadic, et al. [29] reported living with visual impairment affects social relationship, peer acceptance, and functioning in school. At home it affects I and autonomy. They were also concerned about inadequate family support, burden of living with disability, restrictions and emotions.
These issues become more apparent as the child grows into adolescence and adult hood.

Some investigator studied [30-32] the infants treated surgically or with bevacizumab. Morin [30] found higher incidence of NDD who received bevacizumab. However, Kennedy, et al. [31] and Lein, et al. [32] found no adverse effect of treatment with bevacizumab but found combination therapy had a poor outcome.

In our study $90 \%$ of the children treated with bevacizumab were diagnosed to have abnormal development. Although some vision may have been preserved in children who received bevacizumab as rescue therapy, they had poorer neurodevelopmental functional outcome. These varying observations underscore the need for more follow up studies in premature survivors with ROP.

\section{Summary}

This is the first study of neuro developmental disabilities of large number of children with ROP reported from a single center in India a LMIC. In this study population ROP was seen even in infants $>1500$ gm BW and $>32$ wks GA, unlike reports from other developed countries. Treatment of ROP included laser and Bevacizumab therapy. In spite of corrective surgery, even those who had visual correction revealed Visio motor and depth perception abnormalities. Neuro developmental disabilities were found in $68.4 \%$ of ROP affected children. NDD included blindness, cerebral palsy, cognitive, motor, speech and hearing disabilities. Visual impairment was associated with NDD was statistically significant in particular cognitive disability.

\section{Conclusion}

Our study shows that NDDs usually associated with prematurity are further affected by the visual impairments caused by ROP. These findings underscore the importance of prevention of ROP, not only to prevent blindness and visual impairment but also to prevent NDDs. The study emphasizes for pediatricians, to early screening of ROP, and to recognize neurodevelopmental deficiencies and initiate interventional therapy.

\section{Acknowledgement}

We would like to acknowledge the following contributors.

- Marilyn Miller MD, Emeritus Professor of Ophthalmology university of Illinois Eye and Ear Infirmary provided suggestions in early stages of the study.

- Vijayakumar. B, Biostatistician contributed to the data analysis. Aravind Eye Care System, Madurai, Tamil Nadu, India.

- Manish Tandon MD contributed to surgical care of ROP patients. Vitrio Retinal Surgeon, Arvind Eye Care System, Madurai Tamil Nadu, India. 
- Our sincere thanks to Mrs. Chandra for her effort in recruiting and coordination of patients.

\section{Contributors Statement}

Dr. Nagamani Beligere conceptualized and designed the study and was responsible for writing the protocol and conducting neurodevelopmental evaluation of infants included in the study, analyzing the data and drafting the manuscript.

Dr. Vijayalakshmi Perumal Swamy was responsible for conceptualizing and assisted in designing the study, and treating and managing ROP patients, providing patients for the study and contributed to drafting the manuscript.

Jeyaseeli H. Flora, Ophthalmic Vision Rehab.Centre, Arvind Eye Care System, Madurai,Tamil Nadu India,Visual Rehabilitation Specialist and Coordinator helped in communication with the patients in local language.

Marla J. Garska Chicago Light House, Early Intervention Program, North Chicago II (USA) assisted in evaluation of patients.

All authors approved the final manuscripts submitted and agreed.

\section{References}

1. Gilbert C (1997) Retinopathy of prematurity: A global perspective of the epidemics- Population of the babies at risk. Lancet 350: 12-14.

2. Gilbert C, Rahi J, Eckstein M, O'Sullivan J, Foster A (2008) Retinopathy of prematurity in middle-income countries. Implication for control Early Human Develop 84: 77-82.

3. Blencowe $\mathrm{H}$, Lawn JE, Vasquez T, Fielder A, Gilbert $\mathrm{C}$ (2013) Preterm associated visual impairment and estimates of retinopathy of prematurity at regional and global levels for 2010. Pediatric Research 74: 35-49.

4. International Committee for the classification of Retinopathy of Prematurity (2005) The international classification of retinopathy of prematurity revisited. Arch Ophthalmol 123 991-999.

5. Anderson S, Boigon S, Davis K, de Waard C 'Skills Inventory: "Oregon project for preschool children, who are Blind and visually impaired. (6 $6^{\text {th }}$ edn), Sothern Oregon Education service District 101N. Grape St. Medford, Oregon 97501.

6. (2010) (Beery VMI) Beery- Buketencia Developmental Test of Visual Motor Integration sixth edition. Beery K, Buktenica NA et al Modern curriculum pres. Pearson Clinical CO.UK.

7. Vartanian RJ, Besirli CG, Barks JD, Andrews CA, Musch DC (2017) Trends in the screening and treatment of retinopathy of prematurity. Pediatrics 139.

8. Bas AY, Koc E, Dilmen U, ROP Neonatal Study Group (2015) Incidence and severity of retinopathy of prematurity in Turkey.

9. Bas AY, Demirel N, Koc E, Ulubas Isik D, Hirfanoglu IM, et al. (2018) Incidence, risk factors and severity of retinopathy of prematurity in Turkey (TR-ROP study): A prospective, multicenter study in 69 neonatal intensive care units. $\mathrm{Br} \mathrm{J}$ Ophthalmol 102: 1711-1716.

10. Rekha S, Battu RR (1996) Retinopathy of prematurity: Incidence and risk factors. Indian Pediatr 33: 999-1003.
11. Chaudhari S, Patwardhan V, Vaidya U, Kadam S, Kamat A (2009) Retinopathy of prematurity in a tertiary care center--incidence, risk factors and outcome. Indian Pediatr 46: 219-224.

12. Vinekar A, Mangalesh S, Chaitra Jayadev, Gilbert C, Dogra $M$, et al. (2007) Retinopathy of prematurity in Asian Indian babies weighing greater than 1250 grams at birth: Ten-year data from a tertiary care center in a developing country. Indian J Ophthalmol 55: 331-336.

13. Balakrishnan UL, Shaik SMS, Manian NI (2016) Screening based on incidence of severe retinopathy of prematurity in a tertiary care center in India: Are Indian infants different? Int J Contemp Pediatr 3: 847-853.

14. Cook A, White S, Batterbury M, Clark D (2008) Ocular growth and refractive error development in premature infants with or without retinopathy of prematurity. Invest Ophthalmol Vis Sci 49: 5199-5207.

15. Choi MY, Park IK, Yu YSc (2000) Long term refractive outcome in eyes of preterm infants with and without retinopathy of prematurity: Comparison of keratometric value, axial length, anterior chamber depth, and lens thickness.

16. Tasman W (2011) Retinopathy of prematurity: Do we still have problem? The Charles L. Schepens Lecture. Arch Ophthalmol 129: 1083-1086.

17. Kaur S, Sukhija J, Katoch D, Sharma M, Samanta R, et al. (2017) Refractive and ocular biometric profile of children with a history of laser treatment for retinopathy of prematurity. Indian J Ophthalmol 65: 835-840.

18. Kaminer R K, McMahon E (1995) Blindness and visual impairment. Peds in Review 16: 77-78.

19. Msall ME, Phelps DL, DiGaudio KM, Dobson V, Tung B, et al. (2000) Severity of Neonatal Retinopathy of Prematurity Is Predictive of Neurodevelopmental Functional Outcome at Age 5.5 Years. Pediatrics 106: 998-1005.

20. Cooke RW, Foulder-Hughes L, Newsham D, Clarke D (2004) Ophthalmic impairment at 7 years of in children born very preterm. Arch Dis Child Fetal Neonatal Ed. 89: 249253.

21. Schmidt B, Asztalos EV, Roberts RS, Robertson CM, Sauve RS, et al. (2003) Impact of bronchopulmonary dysplasia, brain injury, and severe retinopathy on the outcome of extremely low-birth-weight infants at 18 months: results from the trial of indomethacin prophylaxis in preterms. JAMA 289: 1124-1129.

22. Schmidt B, Davis PG, Asztalos EV, Solimano A, Roberts RS (2014) Research Letter association Between Severe Retinopathy of Prematurity and Nonvisual Disabilities at Age 5 Years. JAMA 311: 523-525.

23. Leversen KT, Sommerfelt K, Rønnestad A, Per Ivar Kaaresen, Theresa Farstad, et al. (2011) Prediction of Neurodevelopmental and Sensory Outcome at 5 Years in Norwegian Children Born Extremely Preterm. Pediatrics 127: e630-e638.

24. Goyen TA, Todd DA, Veddovi M, Wright AL, Flaherty M, et al. (2006) Eye-hand co-ordination skills in very preterm infants < 29 weeks gestation at 3 years: Effects of preterm birth and retinopathy of prematurity. Early Hum Dev 82: 739-745.

25. Beligere N, Perumalswamy V, Tandon M, Mittal A, Floora J, et al. (2015) Retinopathy of prematurity and neurodevelopmental disabilities. Semin Fetal Neonatal Med 20: 345-353.

26. Glass TJA, Chau V, Gardiner J, Foong J, Vinall J, et al. 
(2017) Severe retinopathy of prematurity predicts delayed white matter maturation and poorer neurodevelopment. Arch Dis Child Fetal Neonatal Ed. 102: 532-537.

27. Sveinsdóttir K, Ley D, Hövel H, Fellman V, Hüppi PS, et al. (2018) Relation of Retinopathy of Prematurity to Brain Volumes at Term Equivalent Age and Developmental Outcome at 2 Years of Corrected Age in Very Preterm Infants. Neonatology 14: 46-52.

28. Rainey L, Elsman EBM, van Nispen RMA, van Leeuwen LM, van Rens GHMB (2016) Comprehending the impact of low vision on the lives of children and adolescents; a qualitative approach. Qual Life Res 25: 2633-2643.

29. Tadić V, Hundt GL, Keeley S, Rahi JS; Vision-related Quality of Life (VQoL) group (2015) Seeing it my way: living with childhood onset visual disability. Child Care Health Dev 41: 239-248.

30. Morin J, Luu TM, Superstein R, Ospina LH, Lefebvre F, et al. (2016) Neurodevelopmental Outcomes Following Bevacizumab Injections for Retinopathy of Prematurity. Pediatrics 137.

31. Kennedy KA, Mintz-Hittner HA; BEAT-ROP Cooperative Group (2018) Medical and developmental outcomes of bevacizumab versus laser for retinopathy of prematurity. BEAT-ROP Cooperative Group J AAPOS 22: 61-65.

32. Lein R, Yu MH, Hsu KH, Liao PJ, Chen YP, et al. (2016) Neurodevelopmental Outcomes in Infants with Retinopathy of Prematurity and Bevacizumab Treatment. PLoS One 11: 148019. 